\title{
Multi-objective optimization for creating a low-carbon logistics system through community-based action
}

\author{
Yoshiaki SHIMIZU*, Tatsuhiko SAKAGUCHI ${ }^{*}$ and Hiroki SHIMADA* \\ "Department of Mechanical Engineering, Toyohashi University of Technology \\ 1-1 Hibarigaoka, Tenpaku-cho, Toyohashi 441-8580, Japan \\ E-mail: sakaguchi@me.tut.ac.jp
}

Received 14 April 2015

\begin{abstract}
Against global warming and environmental degradation, it is essential for modern logistics to make sincere efforts to resolve such environmental issues. However, that will likely degrade the economical efficiency under global competition. In fact, since it seems almost impossible to derive a reasonable solution individually, we need to face with the problem as whole society. Deployment of a green logistics incorporated with community-based action is a new paradigm realizing an infrastructure aligned with sustainable development. Noticing importance of such idea, this study concern a green logistics optimization associated with production methods of manufacturers and green attitude of consumers. Actually, for a three-echelon logistics network, we have developed a novel hierarchical method to optimize the production methods with different structures regarding cost and emission of carbon dioxide $\left(\mathrm{CO}_{2}\right)$ at production sites, the available collection center, the paths between members of the logistics network and circular routes over consumers. In this problem, first we aim at minimizing either total cost or $\mathrm{CO}_{2}$ emission through controlling prone and aversion behaviors on sustainability of each logistic member. Then, to integrate these individual problems and evaluate them on the same basis, we have introduced an economic mechanism known as emission trading rate on $\mathrm{CO}_{2}$. Moreover, to discuss the sustainability in a broader logistics system, we note the modal shift in transportation. To show the significance of the proposed approach, we provide a case study and explore some prospects for community-based green logistics.
\end{abstract}

Key words : Sustainability, Community-based logistics optimization, Hybrid meta-heuristic method, Emission trading, Modal shift

\section{Introduction}

Against global warming and environmental degradation, many interests have been paid to the studies popularly termed reverse, closed, green or sustainable logistics (Fiksel, 2010; Nikolopoulou and Ierapetritou, 2012; Srivastava, 2007). Recently, we concerned with a reverse logistics optimization in a practical manner (Shimizu and Sakaguchi, 2013) and deployed an idea for realizing an infrastructure aligned with sustainable development. Actually, we developed a model for green logistics incorporated with co-existence/co-operation of production and consumption (Shimizu, 2014, Nagata, 2014). This study extends such model to discuss on the sustainability through a community-based action that aims at facilitating the low carbon society by developing human-social systems in terms of communicative, institutional and economical approaches.

To work with the problem, we have proposed a community-based model that additionally optimizes the production methods (CLs) with different structures regarding cost and $\mathrm{CO}_{2}$ emission at production sites (PSs) besides popular available collection centers (CCs), paths from PSs to CCs and circular routes travelling client retailers (REs) from every CC. It aims at building a platform where every logistic member in the community can take part in decision-making through mutual understanding about low-carbon production, transportation and consumption. Such mutual learning will eliminate major bottlenecks to shift logistics system from an individual-based action to the community-based one. 
In this development, we particularly note that transportation cost and $\mathrm{CO}_{2}$ emission depend not only on the distance traveled but also on the loading weight. It is practical, therefore, to consider these two factors in parallel or to adopt a bilinear model of distance and load (Weber) or nonlinear one (generalized Weber). Moreover, it had better to consider the fixed operational cost of vehicle for practical accounting. To the best of our knowledge, however, these ideas have been never considered elsewhere associated with vehicle routing problem (VRP) except for our studies (Shimizu, 2011a, 2011b).

The proposed model originally attempts to minimize either total cost or $\mathrm{CO}_{2}$ emission through controlling prone and aversion behaviors on sustainability of each logistic member. As a promising glue to integrate these problems solved individually and to evaluate the decision on the same basis, i.e., cost, we introduce an economic mechanism known as the emission trading for $\mathrm{CO}_{2}$. If manufactures would accept such green tax, and customers also agree with higher price due to the increased logistic cost, we can reduce the $\mathrm{CO}_{2}$ emission eventually. Moreover, to discuss the sustainability from a broader concern, we note a transportation system known as the modal shift that replaces a single transportation mean to multiple ones. Such decision making should be also taken place through community-based actions, and the proposed model is available for such discussion. To show the significance of the proposed approach, we provide a case study with a few plausible scenarios to explore prospects for the community-based logistics.

The rest of the manuscript is organized as follows. Section 2 describes a mathematical formulation of the problem. In Section 3, we explain the proposed hybrid method to solve the problem practically. Discussing some key issues through a case study in Section 4, we will give a conclusion in Section 5.

\section{Problem Formulation}

Taking a global logistics network composed of production site (PS), collection center (CC), and customer or retailer (RE) as shown in Fig. 1, we try to decide the available PSs and their production methods (CLs), paths from PSs to CCs and circular routes from every CC over its client REs. The goal is to provide a practical solution method that enables us to minimize either total cost or $\mathrm{CO}_{2}$ emission. Then, it will be extended so that we can discuss on tradeoff to realize the community-based logistics. In this study, such problem is formulated as the following mixed-integer programming problem under mild assumptions, e.g., round-trip transportation between PS and CC, circular transportation over REs, certainly defined system parameters, etc. Then, we define an objective function of the first problem $f_{1}(\boldsymbol{X})$ called pure cost problem as follows.

$$
\begin{aligned}
f_{1}(X) & =\sum_{i \in I} \sum_{j \in J} T c L_{i j}\left(f 2_{i j}+2 w_{0} t_{i}\right)+\sum_{i \in I i^{\prime} \in I^{\prime}} H p_{i i^{\prime}} f 1_{i i^{\prime}}+\sum_{i \in I} S h 1_{i}\left(\sum_{j \in J} f 2_{i j}\right)+\sum_{j \in J} S h 2_{j}\left(\sum_{i \in I} f 2_{i j}\right) \\
& +\sum_{v \in V} \sum_{p \in P} \sum_{p^{\prime} \in P} c_{v} d_{p p^{\prime}}\left(g_{p p^{\prime} v}+w_{v}\right) z_{p p^{\prime} v}+\sum_{v \in V} F_{v} y_{v}+\sum_{i \in I} \sum_{i^{\prime} \in I} F c_{i i^{\prime}} x_{i i^{\prime}}
\end{aligned}
$$

where $\boldsymbol{X}$ denotes a vector standing for all decision variables shown below. Each term in the right hand side of equation describes the round-trip transportation cost between PS and CC, production cost at PS, shipping cost at PS and $\mathrm{CC}$, circular transportation cost covering every RE and fixed charge for production and vehicle operation, respectively. Just replacing the coefficients of the cost factors in $f_{1}(\boldsymbol{X})$ with those of $\mathrm{CO}_{2}$ emission, we can give the second objective function $f_{2}(\boldsymbol{X})$ to evaluate emission of carbon dioxide.

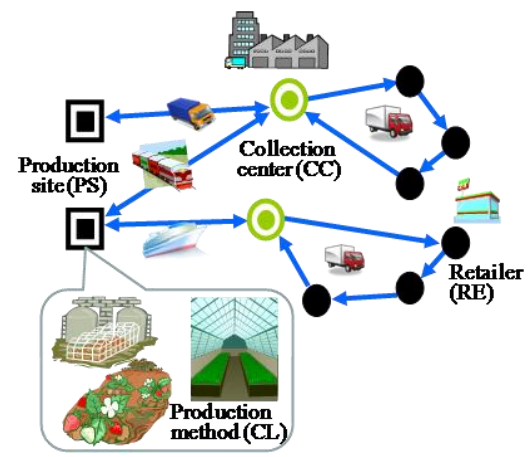

Fig. 1 Global logistics network under consideration: products prodeced differently at production sites are shipped to collection centers by various transportation vehicles. Then they are delivered to every retailer through routing by trucks. 
Since there generally exists a tradeoff between such economy $f_{1}(\boldsymbol{X})$ and environmental issue $f_{2}(\boldsymbol{X})$, the present problem should be resolved through multi-objective optimization. As a promising way to work with it, we introduce a coefficient $\lambda$ associated with the emission trading rate on $\mathrm{CO}_{2}$. Then, we can transform the amount of $\mathrm{CO}_{2}$ emission into the cost (surrogate cost) and finally formulate the following mixed integer programming problem under consideration.

$$
\begin{aligned}
& \text { (p.1) Minimize } f_{1}(\boldsymbol{X})+\lambda f_{2}(\boldsymbol{X}) \\
& \text { subject to } \\
& \sum_{p \in P} z_{k p v} \leq 1, \quad \forall k \in K ; \forall v \in V \\
& \sum_{p^{\prime} \in P} z_{p p^{\prime} v}-\sum_{p^{\prime} \in P} z_{p^{\prime} p v}=0, \quad \forall p \in P ; \forall v \in V \\
& \sum_{j^{\prime} \in J} z_{j j^{\prime} v}=0, \quad \forall j \in J, \forall v \in V \\
& g_{p p^{\prime} v} \leq W_{v} z_{p p^{\prime} v}, \quad \forall p \in P ; \forall p^{\prime} \in P ; \forall v \in V \\
& \sum_{p \in P} \sum_{p^{\prime} \in P} z_{p p^{\prime} v} \leq M y_{v}, \quad \forall v \in V \\
& \sum_{j \in J} \sum_{k \in K} z_{j k v}=y_{v}, \quad \forall v \in V \\
& \sum_{j \in J} \sum_{k \in K} z_{k j v}=y_{v}, \quad \forall v \in V \\
& \sum_{k \in K} g_{k j v}=0, \quad \forall j \in J ; \forall v \in V \\
& \sum_{v \in V} \sum_{p \in P} g_{p k v}-\sum_{v \in V} \sum_{p \in P} g_{k p v}=D_{k}, \quad \forall k \in K \\
& \sum_{p \in P}\left(g_{p k v}-D_{k} z_{p k v}\right)=\sum_{p \in P} g_{k p v}, \forall k \in K, \forall v \in V \\
& \sum_{p \in \Omega} \sum_{p^{\prime} \in \Omega} z_{p p^{\prime} v} \leq|\Omega|-1, \forall \Omega \subseteq P \backslash\{1\},|\Omega| \geq 2, \forall v \in V \\
& \sum_{j \in J} f 2_{i j}=\sum_{i^{\prime} \in I^{\prime}} f 1_{i i^{\prime}}, \quad \forall i \in I \\
& \sum_{i \in I} f 2_{i j}=\sum_{v \in V} \sum_{k \in K} g_{j k v}, \quad \forall j \in J \\
& \sum_{i \in I} f 2_{i j} \leq U_{j}, \quad \forall j \in J \\
& f 1_{i i^{\prime}} \leq S_{i i^{\prime}}^{\max } x_{i i^{\prime}}, \quad \forall i \in I, \forall i^{\prime} \in I^{\prime} \\
& P_{i}^{\mathrm{min}} t_{i} \leq \sum_{j \in J} f 2_{i j} \leq P_{i}^{\mathrm{max}} t_{i}, \quad \forall i \in I \\
& x_{i i^{\prime}} \in\{0,1\}, \forall i \in I, \forall i^{\prime} \in I^{\prime} ; y_{v} \in\{0,1\}, \forall v \in V ; t_{i} \in\{0,1\}, \forall i \in I ; \\
& z_{p p^{\prime} v} \in\{0,1\}, \forall p \in P, \forall p^{\prime} \in P, \forall v \in V \\
& f 2_{i j} \geq 0, \forall i \in I, \forall j \in J ; \quad g_{p p^{\prime} v} \geq 0, \forall p \in P, \forall p^{\prime} \in P, \forall v \in V
\end{aligned}
$$

\section{$\underline{\text { Variables }}$}

$f 1_{i i}$ : amount of production at PS $i$ by production method $i$,

$f 2_{i j}$ : shipping amount from PS $i$ to $\mathrm{CC} j$

$g_{p p^{\prime} v}(t)$ : load of vehicle $v$ on the path from $p \in P$ to $p^{\prime} \in P$

$t_{i}=1$ if PS $i$ is selected; otherwise 0

$x_{i i}=$ production method $i$ ' is selected at PS $i$; otherwise 0 
$y_{v}(t)=1$ if vehicle $v$ is used; otherwise 0

$z_{p p^{\prime} v}(t)=1$ if vehicle $v$ travels on the path from $p \in P$ to $p^{\prime} \in P$; otherwise 0

Parameters

$c_{v}$ : transportation cost per unit load per unit distance of vehicle $v$

$D_{k}$ : demand of retailer $k$

$d_{p p^{\prime}}$ : path distance between $p, p^{\prime} \in P$

$F c_{i i}$ : fixed charge to take production method $i$ ' at PS $i$

$F_{v}$ : fixed charge to operate vehicle $v$

$H p_{i i}$ : production cost per unit load at PS $i$ for production method $i$ '

$L_{i j}$ : distance between PS $i$ and $\mathrm{CC} j$

$M$ : auxiliary constant (Large integer number)

$P_{i}^{\text {max }}$ : maximum available amount at PS $i$

$P_{i}^{\min }$ : minimum available amount at PS $i$

$S_{i i}{ }^{\text {max }}$ : maximum production at PS $i$ for production method $i$ '

$S h 1_{i}$ : shipping cost per unit load at PS $i$

$S h 2_{j}$ : shipping cost per unit load at $\mathrm{CC} j$

$T_{c}$ : transportation cost per unit load from PS to CC

$U_{j}$ : maximum capacity at $\mathrm{CC} j$

$w_{0}, w_{v}$ : unladen weight of vehicle used at PS and CC, respectively

$W_{v}$ : maximum capacity of vehicle $v$

$\lambda$ : emission trading rate on $\mathrm{CO}_{2}$

$\underline{\text { Index set }}$

I: PS; I': CL; J: CC; $K$ : RE, $\Omega$ : a subset of the vertices

The objective function of (p.1) is composed of the pure cost and the surrogate one mentioned already. When we are interested only in the pure cost minimization, $f_{1}(\boldsymbol{X})$ becomes the objective function while $\lambda f_{2}(\boldsymbol{X})$ when interested in the surrogate one. On the other hand, constraints mean that Eq.(2): vehicles cannot visit a customer twice; Eq.(3): vehicles entering a certain RE must leave it; Eq.(4): no travel between CCs; Eq.(5): upper bound on loading capacity for vehicle; Eq.(6): each vehicle must travel once on a certain path; Eqs.(7) and (8):each vehicle leaves only one CC and returns there; Eq.(9): vehicles return to the CC with empty; Eq.(10): customer demand is satisfied; Eq.(11): sum of inlet must be greater than that of outlets by the demand; Eq.(12): Sub-tour elimination; Eqs.(13) and (14): material balances at PS and CC, respectively; Eq.(15): holding capacity at CC is upper bounded; Eq.(16): production is upper bounded at PS per method; Eq.(17): total production at PS must be within the prescribed range. Moreover, either binary or non-negative integer condition is put on the decision variables in terms of their properties.

From this problem, we can decide the available PSs and their production methods, paths from PS to CC and circular routes touring every client REs from each CC under the relevant constraints. Due to the high NP-hardness, however, it is almost impossible to solve this problem with practical size by any currently available commercial software. Against this, our hybrid method (Wada and Shimizu, 2006) can successfully solve several variants, including complicated situations resulting from a variety of real-life conditions. This method is not only practical and powerful but also flexible and adaptive to various applications. So, we intended to extend such idea to the present problem.

\section{Multi-level method incorporating multi-depot vehicle routing problem and modified tabu search}

To solve the above problem practically, we have introduced a few ideas and integrated them into the framework of our conventional method. The first one is how to choose the available PSs and their production methods and to decide the paths from PSs to REs via CCs. Here, every path from each CC to REs determines the client REs for each CC. This is equivalently to solve a customer assignment problem. For the pure cost problem, it is described below by a linear programming problem (LP). 
(p.2) $\min \sum_{i \in I} \sum_{j \in J} T c L_{i j} f 2_{i j}+\sum_{i \in I} \sum_{j \in J} c_{v} d_{i j^{\prime}} f 2_{i j}+$

$$
\sum_{i \in I i^{\prime} \in I^{\prime}} H p_{i i^{\prime}} f 1_{i i^{\prime}}+\sum_{i \in I} S h 1_{i}\left(\sum_{j \in J} f 2_{i j}\right)+\sum_{j \in J} S h 2_{j}\left(\sum_{i \in I} f 2_{i j}\right)
$$

subject to

$$
\begin{aligned}
& f 1_{i i^{\prime}} \leq S_{i i^{\prime}}^{\operatorname{mx}}, \quad \forall i \in I, \forall i^{\prime} \in I^{\prime} \\
& P_{i}^{\min } \leq \sum_{j \in J} f 2_{i j} \leq P_{i}^{\max }, \quad \forall i \in I \\
& \sum_{i \in I} f 2_{i j} \leq U_{j}, \quad \forall j \in J \\
& \sum_{j \in J} f 2_{j k}=D_{k}, \quad \forall k \in K \\
& f 1_{i i^{\prime}} \geq 0, \forall i \in I, \forall i^{\prime} \in I^{\prime}, f 2_{j k} \geq 0, \forall j \in J, \forall j \in J
\end{aligned}
$$

Solving the above LP, we can decide the amount of each production method at PS associated with the available paths between PSs and CCs and the client REs of each CC. The assignment by this approach is truly suitable compared with the conventional ones relying on a certain geometric reasoning such as Voronoi diagram (Man et al., 2012), cluster divisions(Şakir and Küçükdeniz, 2009), polar angles between the depot and the customers (Gillett and Miller, 1989), etc. These just claim the rationality qualitatively and neglect every condition appeared in the formulation of (p.1). That is, they never consider the capacity constraints, the handling cost and the practical transportation cost accounting in the objective function. Against this, since the auxiliary problem (p.2) might partly reflect some conditions of (p.1), we can assert its rationality even quantitatively.

Secondly, instead of solving (p.2) directly, we transform it into the equivalent minimum cost flow (MCF) problem by introducing some virtual nodes and edges to the physical structure so that we can reflect some constraints of the present formulation. This graph structure and its label information are shown in Fig.2 and Table 1, respectively. Then, we applied a graph algorithm of MCF problem like RELAX4 (URL1, Accessed in 2013) to solve this problem extremely fast by incorporating its sensitivity analysis. After all, we can speed up the outer-loop search where we need to solve the similar LP repeatedly. This graph is still valid for the problems with other objective functions, i.e., the surrogate cost or the augmented one. It is necessary only to replace the label information regarding the costs with those of the respective problem. Apparently, for every node of CL and PS without inflow in the MCF graph, CL will not be used and PS not be opened, respectively.

Thirdly, letting REs thus allocated as the clients for each CC, we move on the inner-loop search that refers to individual VRP per CC. It should be noted here the Weber basis cost accounting is essential for this VRP to hold consistency when evaluating the transportation cost from PS to CC and that of VRP. Though there are several

\begin{tabular}{|c|c|c|}
\hline Edge (from to) & Cost & Capacity \\
\hline Source - $\sum$ (Dummy) & $-M$ & $\sum_{i \in I} P_{i}^{\min }$ \\
\hline Source - PS(in) $i$ & 0 & $P_{i}^{\max }-P_{i}^{\min }$ \\
\hline$\sum-\mathrm{PS}$ (in) $i$ & 0 & $P_{i}^{\min }$ \\
\hline PS(in) $i-$ CL $i i^{\prime}$ & $H p_{i i}$ & $S_{i i}{ }^{\max }$ \\
\hline CL $i i^{\prime}-$ PS(out) $i$ & 0 & $S_{i i}{ }^{\max }$ \\
\hline PS(out) $i-\mathrm{CC} j$ & $T \mathrm{c} L i j+$ Ship $1_{i}$ & $P_{i}^{\max }$ \\
\hline Between double nodes of $\mathrm{CC} j$ & Ship $2_{j}$ & $U_{j}$ \\
\hline $\mathrm{CC} j-\mathrm{RE} k$ & $c_{v} d_{j k}$ & $D_{j}$ \\
\hline RE $k$ - Sink & 0 & $D_{k}$ \\
\hline
\end{tabular}
methods to provide a good approximated solution of VRP like saving method (Clarke and Wright, 1964), none of the conventional methods adopt the Weber basis. Against this, our Weber basis saving method is conveniently applied to derive an initial solution for this aim. Its algorithm is outlined as follows.

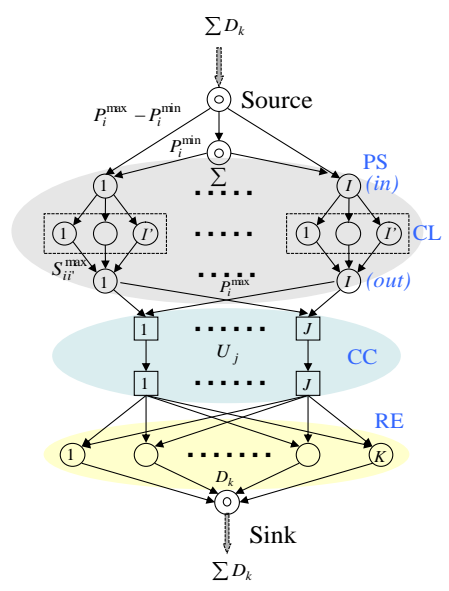

Table 1 Labeling on the edge for MCF graph

Fig. 2 MCF graph structure: virtual nodes and edges are added to satisfy the prescribed conditions of the model. 


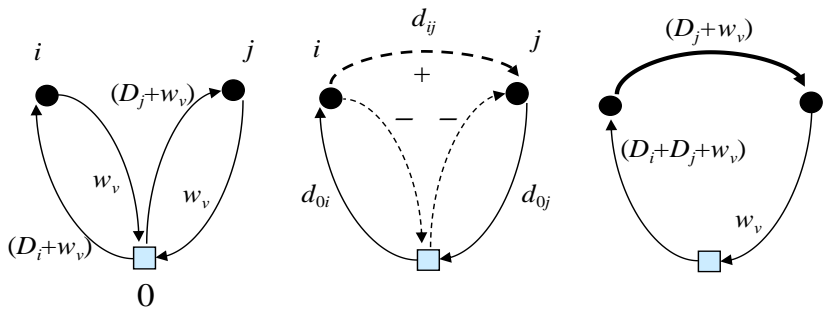

Fig. 3 Scheme to compute Weber basis saving value: the round trip (left) may be merged (right) after evaluating(middle) the saving value.

Step 1: Create a round trip route from every CC to each RE. Then, compute the Weber basis saving value by $s_{i j}=\left(d_{0 j}-d_{0 i}-d_{i j}\right) D_{j}+\left(d_{0 j}+d_{i 0}-d_{i j}\right) w_{v} \quad$ (Refer to Fig.3). Sort the savings in descending order and store them in the saving list.

Step 2: Starting from the top of the saving list, determine whether there exist two routes, one containing $(i, 0)$ and the other containing $(0, j)$. If so, merge the route as long as the combined demand is less than the vehicle capacity and the saving is greater than $-F_{v} / c_{v}$. Within this range, we can save the additional expense due to another fixed cost of vehicle.

Since the Weber basis saving method derives only an approximated solution, we attempt to improve it through the modified tabu search. The original tabu search (Glover, 1989) is a simple but powerful heuristic method that refers to a local search with a certain memory structure. In the present local search, we randomly generate a neighbor solution by either of insert, swap or 2-opt operations for the cases between the routes (extra-route) and within the route (intra-route). These operations of the extra-loop are shown in Fig. 4. To avoid trapping into a local minimum, our modified method allows even a degraded neighbor solution to be a new tentative solution as long as it would be feasible and not be involved in the tabu lists. Such decision is made in terms of the probability $p$ whose distribution obeys the following Maxwell-Boltzmann function used in simulated annealing (Kirkpatrick et al., 1983).

$$
p=\left\{\begin{array}{cl}
1 & \text { if } \Delta e \leq 0 \\
\exp (-\Delta e / T) & \text { if } \Delta e<\varepsilon . \\
0 & \text { if } \Delta e>\varepsilon
\end{array}\right.
$$

where $\Delta e$ denotes the difference of objective function from the tentative best value, and $\varepsilon$ a small positive number. Moreover, $T$ is the temperature that will decrease along with the iteration $k$ geometrically, i.e., $T^{k}=\sigma T^{k-1}, \sigma<1$.

Thus derived result is accumulated over the network and the resulting total evaluation is fed back to update the first level problem. Fourthly, such outer-loop search is also preceded by the modified tabu search mentioned above. This time, however, its neighbor solution is randomly generated by either of flip/flop or swap operation for the element(s) of binary sequence that represents the available PSs and CLs as illustrated in Fig. 5. In a summary, the outline of this algorithm is shown in Fig.6.

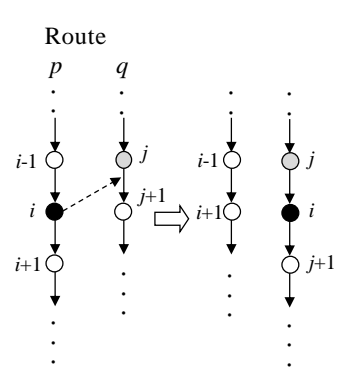

(a) Insertion

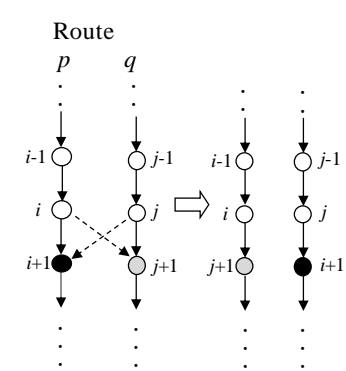

(b) Exchange

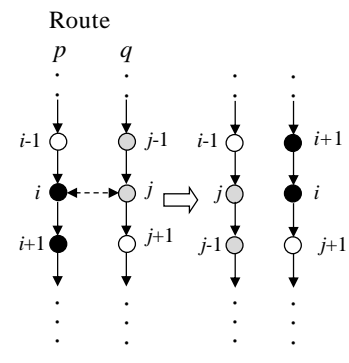

(c) 2-opt

Fig. 4 Scheme to generate neighbor solution in inner-loop search: either of insert, swap or 2-opt operations are randomly selected for the extra-route update and intra-loop one. 


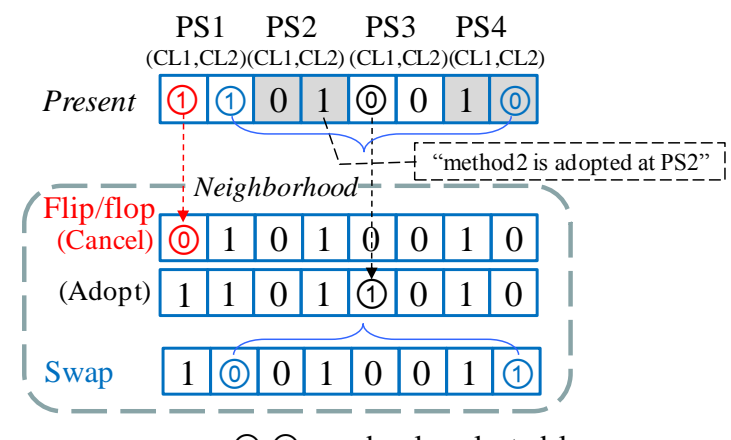

(0), (1): randomly selected locus

Fig. 5 Scheme to generate neighbor solution in outer-loop search: this is depicted for the case with $|\mathrm{I}|=4$ and $\left|\mathrm{I}^{\prime}\right|=2$. Present status is changed by either of flip/flop or swap operation for the element(s) of binary sequence that represents the available PSs and CLs

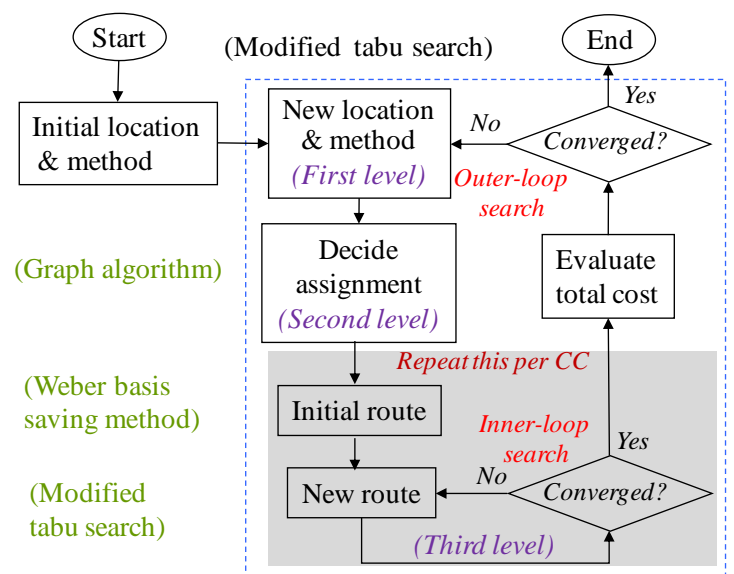

Fig. 6 Outline of the algorithm. It refers to three level procedure composed of graph algorithm, Weber basis saving method and modified tabu search.

\section{Numerical experiments and discussion}

\subsection{Preliminary experiments}

To examine a solution ability of the proposed method, we prepared a small cost minimization problem with randomly generated system parameters within the prescribed ranges and like $|\mathrm{I}|=3,\left|\mathrm{I}^{\prime}\right|=2,|\mathrm{~J}|=2$ and $|\mathrm{K}|=10$. Then we solved it both by the proposed method and a commercial solver termed CPLEX. Since multiple assignments are allowed in the MCF problem formulation, both results illustrated in Fig.7were not exactly the same. However, gap of the objective value from CPLEX stayed only within small level (2.17\%). Noticing possible number of combination maximally reaches at $2^{6} \cdot 10 !=2.32 \times 10^{8}$ even for such small problem, we can claim the high solution ability of the proposed method. Moreover, we can easily suppose it becomes impossible to solve larger problems by CPLEX due to the exponential increase in combinations.

Against this, to show the proposed method can cope with the larger size problems, we provided the benchmark problems by generating their system parameters within the prescribed ranges. We summarized the results in Table 2 on the basis of the averaged value of five trials. Thereat, column of "Problem size" shows the number of PS, CC, RE and CL, i.e., $|\mathrm{I}|,|\mathrm{J}|,|\mathrm{K}|$ and $\left|\mathrm{I}^{\prime}\right|$, respectively. On the other hand, from the improved rate (IMP.RATE) defined by $\left(f^{*}-f_{1}\right) / f_{1}$, we know pretty improvements are given by the proposed method. Here, $f^{*}$ and $f_{1}$ denote the first and converged objective function values, respectively. As supposed a priori, computation time (CPU) expands rapidly along with the problem size. In a summary, we can confirm the solution ability of the proposed method even for the problems with practical sizes. 


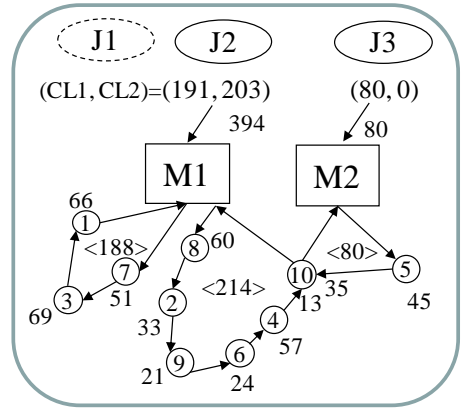

Proposed: $f_{1}=545,828$

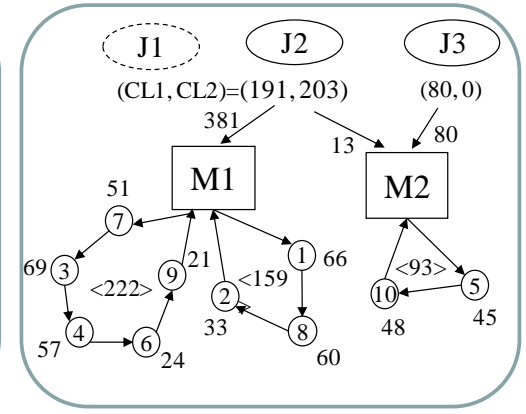

CPLEX: $f_{1}=534,233$

Fig. 7 Comparison of the results for the sample problem between the proposed method and CPLEX: J1-J3 denote PSs, M1 and M2 CCs and circled numbers REs, respectively. The other figures show the amounts of product produced at each PS, collected at CC and delivered to RE (demand). From these values, we confirmed every capacity constraint is satisfied regarding PSs, CCs and trucks. In this case, multiple assignment is occurred at RE-10.

Table 2 Result of benchmark problems

\begin{tabular}{c|r|r|r|r}
\hline \hline Problem size & \multicolumn{1}{c|}{ COST $[¥]$} & IMP.RATE & Iteration (Outer loop) & \multicolumn{1}{c}{ CPU [s] } \\
\hline$(5,3,20,2)$ & 683663.0 & 0.316 & 496.0 & 1.030 \\
\hline$(10,5,50,2)$ & 1436566.3 & 0.237 & 1600.0 & 14.294 \\
\hline$(10,10,100,2)$ & 2092999.0 & 0.357 & 1612.0 & 51.622 \\
\hline
\end{tabular}

\subsection{Case study}

We carried out a case study incorporated with the Google map API to retrieve the real distance data from address of the locations and draw the resulting routes on the map. Adding some necessary information for the present concern, we built a model for the case study that concerns grocery logistics supplying tomato in Nagoya city in Japan (Nakajima et al., 2011). First, we outline the description of the model under consideration below.

1. 3 production sites located in Kumamoto area (PS1; far from Nagoya), Gifu (PS2; very near) and Tahara (PS3; near)

2. 3 collection centers located in Nagoya $(\mathrm{CC} 1, \mathrm{CC} 2, \mathrm{CC} 3)$

3. 17 super markets located in Nagoya as retailers (RE1 - RE17)

4. 2 production methods at production sites (CL1=ground, CL2=in house)

5. 3 scenarios on transportation media listed in Table 3

6. Distances between the members of logistics are retrieved in terms of Google map API.

7. Various parameters listed in Tables 4-6 are collected from literatures (URL2-URL7) as much as possible except for someone assumed adequately.

We summarize the results of the pure cost and the surrogate cost $\left(\mathrm{CO}_{2}\right.$ emission) minimization problems under the prescribed scenarios in Tables 7 and 8, respectively. The secondary objective function of each problem i.e., $\mathrm{CO}_{2}$ emission in Table 7 and total cost in Table 8, respectively is evaluated by the respective optimal solution. Middle part of the tables shows the trading scheme between the production sites and collection centers, i.e., the resulting path from PS to CC and production method at PS for shipping product. Moreover, bottom part of the tables describes the results for VRP for each CC. As supposed beforehand, we obtained the different decisions depending on the adopted objective function and scenarios. Here, we know the effect of the modal shift to rail or sea transportation both regarding the cost and $\mathrm{CO}_{2}$ emission. Under the present parameter setting, scenario 2 (multi-modal rail) seems to be the best result for this logistic system, i.e., gained the minimum values at both objective functions among the scenarios.

In particular, we can observe the trade-off relation between the results of cost and $\mathrm{CO}_{2}$ emission minimization problems under every scenario. So it is meaningful to consider these two objectives at the same time to lead a decision from more total viewpoint. Actually, as mentioned already, we integrated these two objectives through the emission trading rate and solved the aimed problem (p.1). We summarized the results under every scenario in Fig.8. Apparently, the augmented total cost increases along with the emission trading rate in every case. On the other hand, 
$\mathrm{CO}_{2}$ emission is kept constant by a certain point where we have the same optimal solution as that of the pure cost minimization problem. Against this, when the emission trading rate for each scenario becomes greater than 37,22 and $28\left[¥ / \mathrm{kg}-\mathrm{CO}_{2}\right]$, respectively, we know it possible to decrease $\mathrm{CO}_{2}$ emission. Thereat, the optimal solutions are changed so as to increase the available amount of product at site PS1 by ground made.

Though those critical emission trading rates are too high compared with the value that is presently viewed plausible in Japan, say 2 [¥/kg- $\left.\mathrm{CO}_{2}\right]$ (Kakumoto and Irohara, 2010), they are comparable to those applied in a few countries, i.e., Sweden: 17.13, Norway: 18.86 and Italy: 100.26 (Japanese ministry of the environment, 2000). We could discuss this issue more generally and certainly if the proposed idea is applied in terms of more reliable parameters and deployed in more global and total logistics systems. However, this study, as it is, has a great significance to show the possibility of reducing the $\mathrm{CO}_{2}$ emission by controlling the emission trading rate. To facilitate such movement in advance, earnest efforts that make turn consumption behavior to the eco-efficient products and shift to multi-modal transportation become essential through mutual understanding about the community-based actions. The proposed approach may contribute to provide some successful scenarios for such community-based actions among the logistics members.

Table 3 Supposed scenarios on transportation media

\begin{tabular}{l|r|r|r}
\hline \hline Production site & PS1; Kumamoto (far from Nagoya) & PS2;Gifu (very near) & PS3;Tahara (near) \\
\hline Scenario 1 & 15ton Truck & 4ton Truck & 10ton Truck \\
\hline Scenario 2 & Rail + 4ton Truck (multi-modal) & 4ton Truck & 10ton Truck \\
\hline Scenario 3 & Ferry + 4ton Truck (multi-modal) & 4ton Truck & 10ton Truck \\
\hline
\end{tabular}

Table 4 Parameter setting at production site

\begin{tabular}{|c|c|c|c|c|c|c|c|}
\hline \multicolumn{2}{|c|}{ Production site } & \multicolumn{2}{|c|}{ PS1 } & \multicolumn{2}{|c|}{ PS2 } & \multicolumn{2}{|c|}{ PS3 } \\
\hline \multirow{2}{*}{ Shipping amount $[\mathrm{kg}]$} & Upper & & 65300 & & 73007 & & 86700 \\
\hline & Lower & & 32700 & & 36900 & & 43400 \\
\hline \multicolumn{2}{|l|}{ Shipping cost $[¥ / t]$} & & 24150 & & 29000 & & 31000 \\
\hline \multicolumn{2}{|c|}{ Emission from shipping $\left[\mathrm{Kg}-\mathrm{CO}_{2} / \mathrm{t}\right]$} & & 20 & & 40 & & 30 \\
\hline \multicolumn{2}{|l|}{ Production method } & Ground & House & Ground & House & Ground & House \\
\hline \multirow[t]{2}{*}{ Production cost $[¥ / \mathrm{t}]$} & Operating & 65370 & 103890 & 63000 & 123000 & 58000 & 98000 \\
\hline & Fixed-charge & 45950 & 103890 & 63000 & 77240 & 58000 & 98000 \\
\hline \multicolumn{2}{|c|}{ Available production $[\mathrm{Kg}]$} & 4000 & 1500 & 4500 & 1800 & 4000 & 3500 \\
\hline \multicolumn{2}{|c|}{ Emission by production $\left[\mathrm{Kg}-\mathrm{CO}_{2} / \mathrm{t}\right]$} & 354 & 640 & 572 & 872 & 790 & 408 \\
\hline
\end{tabular}

Table 5 Parameter setting at market

\begin{tabular}{r|r|r|r}
\hline \hline & Available capacity $[\mathrm{Kg}]$ & Shipping cost $[¥ / \mathrm{t}]$ & Emission $\left[\mathrm{Kg}-\mathrm{CO}_{2} / \mathrm{t}\right]$ \\
\hline $\mathrm{CC} 1$ & 7370 & 6400 & 30 \\
\hline $\mathrm{CC} 2$ & 6530 & 5120 & 24 \\
\hline CC 3 & 8670 & 6800 & 20 \\
\hline
\end{tabular}

Table 6 Parameter setting for vehicle

\begin{tabular}{|c|c|c|c|c|c|}
\hline \multirow{2}{*}{ Medium } & \multicolumn{3}{|c|}{ Truck payload } & \multirow{2}{*}{ Rail } & \multirow{2}{*}{ Ferry } \\
\hline & 4 ton & 10 ton & 15 ton & & \\
\hline Trans. cost unit $[¥ / \mathrm{t} \cdot \mathrm{km}]$ & 35.7 & 21.3 & 16.4 & 10.4 & 11.6 \\
\hline Fixed charge [¥] & 12000 & 18000 & 22000 & 22000 & 17000 \\
\hline $\mathrm{CO}_{2}$ emission unit $\left[\mathrm{Kg}-\mathrm{CO}_{2} / \mathrm{t} \cdot \mathrm{km}\right]$ & 0.177 & 0.132 & 0.11 & 0.022 & 0.04 \\
\hline
\end{tabular}

\section{Conclusion}

Provision for essential infrastructure aligned with sustainable development is urging us to realize green logistics incorporating co-existence of manufacturers and consumers. This paper has concerned the green logistics optimization associated with the community-based action that will facilitate mutual understanding about low-carbon production, 
transportation and consumption and eliminate major bottlenecks to consider the problem as a whole. Actually, taking a multi-layer logistics network, we have developed a novel approach to optimize the augmented cost comprised the pure economic cost and the surrogate one referring to $\mathrm{CO}_{2}$ emission. Through a case study under some plausible scenarios associated with the modal shift, we have shown the significance of the proposed approach and explored some prospects for green logistics in global society.

Table 7 Results of pure cost minimization problem

\begin{tabular}{|c|c|c|c|c|}
\hline \multicolumn{2}{|c|}{ Scenario \# } & "1 (only Truck) & 2 (multi modal: Rail) & 3 (multi modal: Ferry) \\
\hline \multirow{2}{*}{$\begin{array}{l}\text { Objective } \\
\text { value }\end{array}$} & Pure cost & 1298496.1 [¥] & $1290265.46[¥]$ & 1292969.87[¥] \\
\hline & $\mathrm{CO}_{2}$ emission & $7377.621\left[\mathrm{Kg}-\mathrm{CO}_{2}\right]$ & $7232.351\left[\mathrm{Kg}-\mathrm{CO}_{2}\right]$ & $7305.981\left[\mathrm{Kg}-\mathrm{CO}_{2}\right]$ \\
\hline \multicolumn{2}{|c|}{$\begin{array}{l}\text { Path from PS to CC } \\
\& \text { Production method }\end{array}$} & $\begin{array}{l}(\mathrm{PS} 1, \text { Ground }) \rightarrow(\mathrm{CC} 1)^{* 1} \\
(\mathrm{PS} 2, \text { Ground }) \\
\quad \rightarrow(\mathrm{CC} 1, \mathrm{CC} 2) \\
(\mathrm{PS} 3, \text { Ground }) \rightarrow(\mathrm{CC} 2)\end{array}$ & $\begin{array}{l}\text { (PS1, Ground }) \rightarrow(\mathrm{CC} 2) \\
(\mathrm{PS} 2, \text { Ground }) \\
\quad \rightarrow(\mathrm{CC} 1, \mathrm{CC} 2) \\
(\mathrm{PS} 3, \text { Ground }) \rightarrow(\mathrm{CC} 2)\end{array}$ & $\begin{array}{l}\text { (PS1, Ground }) \rightarrow(\mathrm{CC} 1) \\
(\mathrm{PS} 2, \text { Ground }) \\
\quad \rightarrow(\mathrm{CC} 2, \mathrm{CC} 1) \\
(\mathrm{PS} 3, \text { Ground }) \rightarrow(\mathrm{CC} 2)\end{array}$ \\
\hline \multirow{3}{*}{$\begin{array}{l}\text { Route } \\
\text { over REs }\end{array}$} & From CC1 & $\begin{array}{l}\mathrm{V} 1: 14 \rightarrow 5 \rightarrow 2 \rightarrow 1 \rightarrow 11^{* 2} \\
\mathrm{~V} 2: \\
8 \rightarrow 17 \rightarrow 16 \rightarrow 7 \rightarrow 12 \rightarrow 13\end{array}$ & $\begin{array}{l}\mathrm{V} 1: 14 \rightarrow 5 \rightarrow 2 \rightarrow 1 \rightarrow 11 \\
\mathrm{~V} 2: \\
8 \rightarrow 17 \rightarrow 16 \rightarrow 7 \rightarrow 12 \rightarrow 13\end{array}$ & $\begin{array}{l}\mathrm{V} 1: 14 \rightarrow 5 \rightarrow 2 \rightarrow 1 \rightarrow 11 \\
\mathrm{~V} 2: \\
8 \rightarrow 17 \rightarrow 16 \rightarrow 7 \rightarrow 12 \rightarrow 13\end{array}$ \\
\hline & From CC2 & $\begin{array}{l}\text { V1: } 15 \rightarrow 3 \rightarrow 10 \rightarrow 9 \\
\quad \rightarrow 4 \rightarrow 6 \rightarrow 11\end{array}$ & $\begin{array}{l}\mathrm{V} 1: 15 \rightarrow 3 \rightarrow 10 \rightarrow 9 \\
\quad \rightarrow 4 \rightarrow 6 \rightarrow 11\end{array}$ & $\begin{array}{l}\mathrm{V} 1: 15 \rightarrow 3 \rightarrow 10 \rightarrow 9 \\
\quad \rightarrow 4 \rightarrow 6 \rightarrow 11\end{array}$ \\
\hline & From CC3 & N/A ${ }^{* 3}$ & N/A & N/A \\
\hline
\end{tabular}

${ }^{* 1}$ Product produced at PS1 by ground is shipped to collection center $\mathrm{CC} 1$.

*2 Vehicle V1 departing from CC1 visits customer 14 first and move on the rests in turn.

*3 No shipping from CC3 in this scenario.

Table 8 Results of $\mathrm{CO}_{2}$ emission minimization problem

\begin{tabular}{|c|c|c|c|c|}
\hline \multicolumn{2}{|c|}{ Scenario \# } & 1 (only Truck) & 2 (multi modal: Rail) & 3 (multi modal: Ferry) \\
\hline \multirow{2}{*}{$\begin{array}{l}\text { Objective } \\
\text { value }\end{array}$} & $\mathrm{CO}_{2}$ emission & $5602.88\left[\mathrm{Kg}-\mathrm{CO}_{2}\right]$ & $5321.76\left[\mathrm{Kg}-\mathrm{CO}_{2}\right]$ & $5463.76\left[\mathrm{Kg}-\mathrm{CO}_{2}\right]$ \\
\hline & Pure cost & $1528255[¥]$ & $1513349[¥]$ & $1517790[¥]$ \\
\hline \multicolumn{2}{|c|}{$\begin{array}{l}\text { Path from PS to CC } \\
\& \text { Production method }\end{array}$} & $\begin{array}{l}(\mathrm{PS} 1, \text { Ground }) \rightarrow(\mathrm{CC} 3) \\
(\mathrm{PS} 2, \text { Ground }) \\
\quad \rightarrow(\mathrm{CC} 1, \mathrm{CC} 3) \\
(\mathrm{PS} 3, \text { House }) \rightarrow(\mathrm{CC} 3)\end{array}$ & $\begin{array}{c}(\mathrm{PS} 1, \text { Ground }) \rightarrow(\mathrm{CC} 3) \\
(\mathrm{PS} 2, \text { Ground }) \\
\rightarrow(\mathrm{CC} 1, \mathrm{CC} 3) \\
(\mathrm{PS} 3, \text { House }) \rightarrow(\mathrm{CC} 3)\end{array}$ & $\begin{array}{c}(\mathrm{PS} 1, \text { Ground }) \rightarrow(\mathrm{CC} 3) \\
(\mathrm{PS} 2, \text { Ground }) \\
\rightarrow(\mathrm{CC} 1, \mathrm{CC} 3) \\
(\mathrm{PS} 3, \text { House }) \rightarrow(\mathrm{CC} 3)\end{array}$ \\
\hline \multirow[b]{3}{*}{$\begin{array}{l}\text { Route } \\
\text { over REs }\end{array}$} & From CC1 & $\mathrm{V} 1: 3 \rightarrow 10 \rightarrow 15$ & $\mathrm{~V} 1: 11 \rightarrow 4 \rightarrow 9 \rightarrow 10 \rightarrow 15$ & $\mathrm{~V} 1: 4 \rightarrow 9 \rightarrow 10 \rightarrow 15$ \\
\hline & From $\mathrm{CC} 2$ & N/A & N/A & N/A \\
\hline & From CC3 & $\begin{array}{l}\text { V1: } \\
17 \rightarrow 8 \rightarrow 4 \rightarrow 9 \rightarrow 6 \rightarrow 15 \rightarrow 11 \\
\text { V2: } 5 \rightarrow 2 \rightarrow 1 \\
\text { V3: } 16 \rightarrow 7 \rightarrow 12 \rightarrow 13 \rightarrow 14\end{array}$ & $\begin{array}{l}\text { V1: } \\
5 \rightarrow 6 \rightarrow 15 \rightarrow 3 \rightarrow 10 \rightarrow 14 \\
\text { V2: } 2 \rightarrow 1 \\
\text { V3: } \\
16 \rightarrow 17 \rightarrow 8 \rightarrow 13 \rightarrow 12 \rightarrow 7\end{array}$ & $\begin{array}{l}\text { V1: } \\
8 \rightarrow 17 \rightarrow 7 \rightarrow 13 \rightarrow 12 \rightarrow 16 \\
\text { V2: } 2 \rightarrow 1 \\
\quad \text { V3: } 5 \rightarrow 14 \rightarrow 15 \rightarrow 6 \\
\quad \rightarrow 15 \rightarrow 3 \rightarrow 10 \rightarrow 11\end{array}$ \\
\hline
\end{tabular}

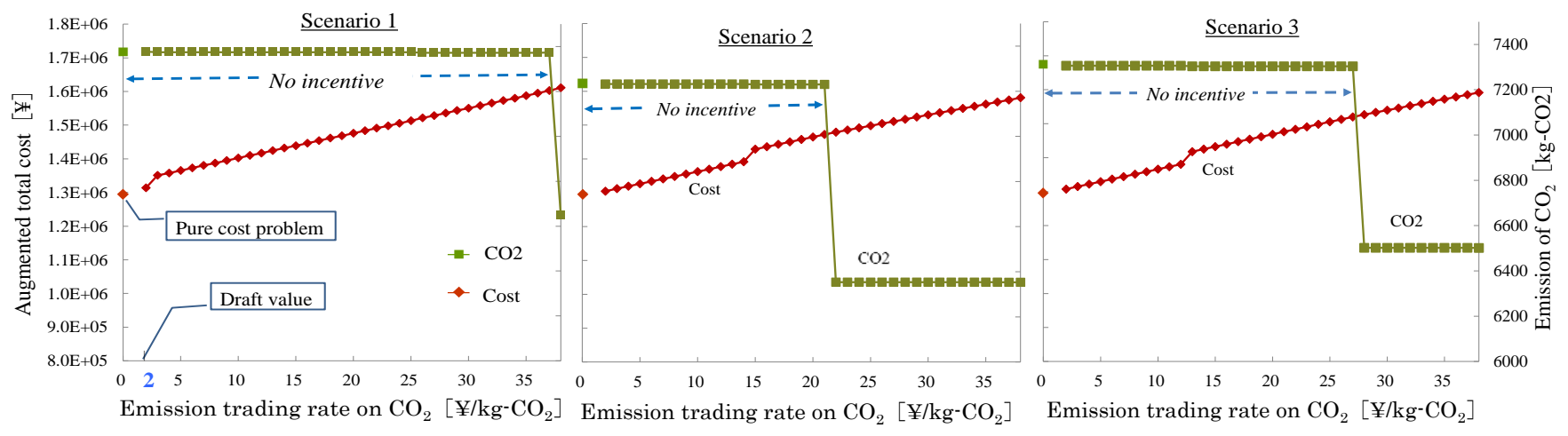

Fig. 8 Trend of two factors in objective function with emission tradeing rate under different scenarios: Scenario 1: uni-modal (only Truck), Scenario 2: multi-modal (Rail), Scenario 2: multi-modal (Ferry); when the emission trading rate for each scenario becomes greater than 37,22 and $28\left[¥ / \mathrm{kg}-\mathrm{CO}_{2}\right]$, respectively, we know it possible to decrease $\mathrm{CO}_{2}$ emission. 
Future studies should be devoted to establish a total decision support system associated with a generic multi-objective optimization. Various real-world applications while extending the system boundary are also meaningful to accumulate the wisdom toward the sustainable development.

\section{Acknowledgements}

This research was partially supported by the Ministry of Education, Science, Sports and Culture, Grant-in-Aid, 25350442, Japan and a project of RISTEX in JST (Community-Based Actions against Global Warming and Environmental Degradation)

\section{References}

Clarke, G., Wright, J., "Scheduling of vehicles from a central depot to a number of delivery points". Operations Research, vol.12, no.4, pp.568-581, 1964.

Fiksel, J.,” Evaluating Supply Chain Sustainability”. CEP, no.5, pp.28-36, 2010.

Gillett, B.E., Miller, L. R., "A heuristic algorithm for the vehicle dispatch problem". Operations Research, vol.22, pp.340-349, 1974.

Glover, F., “Tabu search-part I”. ORSA Journal on Computing, vo.1, no.3, pp.190-206, 1989.

Kakumoto, K., Irohara, T., "Optimization and Sensitivity Analysis of an International Transportation Scheduling Problem Incorporating $\mathrm{CO}_{2}$ Emission Levels". J Jpn Ind. Manage. Assoc., vol.61, no.2, pp.46-53, 2010 (in Japanese).

Kirkpatrick S., Gelatt, C.D, Vecch, M.P., "Optimization by Simulated Annealing”, Science, vol.220, pp.671-680, 1983.

Man,Q., Mingyi, D., Liu, Y., "Application of Voronoi diagrams and multiangle measurable image in the urban POI location and site generation". Proc. Information Science and Control Engineering 2012 (ICISCE 2012), pp. 1 - 5, Shenzhen (7-9 Dec., 2012).

Nagata, J. (ed), " Creating a low-carbon production, retail \& shopping system for Nagoya". Annual report of RISTEX, 2014 (in Japanese).

Nakajima, H., Ohno, T., Ikemori, F., Takagi, K., "Estimate by LCA technique of $\mathrm{CO}_{2}$ exhaust according to vegetables and fruits consumption of Nagoya City". Annual report of Nagoya City Environmental Science Research Institute, no.41, pp.15-19, 2011 (in Japanese).

Nikolopoulou, A., Ierapetritou, M. G., "Optimal design of sustainable chemical processes and supply chains: A review". Computers and Chemical Engineering, vol.44, no.9, pp.94-103, 2012.

Şakir, E., Küçükdeniz, T., "A fuzzy clustering-based hybrid method for a multi-facility location problem”. Journal of Intelligent Manufacturing, vol.20, no.2, pp.259-26, 2009.

Shimizu, Y., "Advanced Saving Method to Evaluate Economic Concern". Transactions of the Institute of Systems, Control and Information Engineers, vol.24, no.2, pp.39-41, 2011a (in Japanese).

Shimizu, Y., "A Meta-heuristic Approach for Variants of VRP in Terms of Generalized Saving Method". Transactions of the Institute of Systems, Control and Information Engineers, vol.24, no.12, pp.287-295, 2011b (in Japanese).

Shimizu, Y., Sakaguchi. T., "Generalized Vehicle Routing Problem for Reverse Logistics Aiming at Low Carbon Transportation”. Industrial Engineering \& Management Systems, vol.12, no.2, pp.161-170, 2013.

Shimizu, Y., "Incorporating Green into Production and Consumption Behaviours toward Sustainable Logistics Optimization in Process Systems". 24 ${ }^{\text {nd }}$ European Symposium on Computer Aided Process Engineering, Budapest, Hungary (15-18 June, 2014).

Srivastava, S., "Green Supply-chain Management: A State-of-art Literature Review". International Journal of Management Reviews, vol.9, pp.53-80, 2007.

Wada, T., Shimizu, Y., "A Hybrid metaheuristic approach for optimal design of total supply chain network". Transactions of the Institute of Systems, Control and Information Engineers, vol.19, no.2, pp.69-77, 2006 (in Japanese).

Japanese ministry of the environment: White paper (general view), 2000

URL1, Massachusetts Institute of Technology, Lab. for information and decision systems (online), available from $<$ http://mit.edu/dimitrib/www/home.html>, (accessed on 27 October, 2015).

URL2, Statics of production states for vegetables and fruits, Ministry of Agriculture, Forestry and Fisheries (online), available from $<$ http://www.e-stat.go.jp/SG1/estat/List.do?lid=000001061833>, (accessed on 27 October, 2015).

URL3, Report on cost accounting for food logistics, Ministry of Agriculture, Forestry and Fisheries (online), available from <http://www.e-stat.go.jp/SG1/estat/List.do?lid=000001112277>, (accessed on 27 October, 2015).

URL4, Guideline to compute CO2 emission in logistics, Ministry of Economy, Trade and Industry $\cdot$ Minister of Land, Infrastructure, Transport and Tourism (online), available from 
$<$ http://www.enecho.meti.go.jp/policy/images/060518pamph.pdf>, (accessed on 14 April, 2015).

URL5, Database of $\mathrm{CO} 2$ emission rate on food materials, Environmental report of Ajinomoto Inc. (online), available from < http://www.ajinomoto.com/jp/activity/environment/pdf/2010/lcco2.pdf >, (accessed on 27 October, 2015).

URL6, White Paper on the environment of Tokyo Metropolitan Central Wholesale Market (online), available from $<$ http://www.shijou.metro.tokyo.jp/gyosei/04/04.html>, (accessed on 27 October, 2015).

URL7, Report on the fair of business transportation in Osaka city (online), available from < http://www.city.osaka.lg.jp/port/cmsfiles/contents/0000002/2591/12.pdf>, (accessed on 14 April, 2015). 